\title{
El delirio de la palabra: el retorno a la vida de Robert Antelme
}

\author{
Inés VALLE MORÁN \\ Departamento de Historia Contemporánea \\ Universidad Complutense de Madrid \\ ines_va@hotmail.com
}

\begin{abstract}
RESUMEN
En mayo de 1945, tras la liberación de Dachau, Robert Antelme, miembro de la resistencia francesa, comenzó su complejo proceso de vuelta a la vida. Dos años después, publicó su única obra, L'espèce humaine, el relato de su experiencia en el campo. Desde entonces, no volvió a hablar ni a escribir sobre su pasado, no concedió entrevistas, ni se ha publicado ninguna biografía suya. Este artículo considera el período inmediatamente posterior a su retorno en el que se vio asaltado por el «delirio de la palabra». Lo singular de este caso es que contamos con testimonios de familiares y amigos -Marguerite Duras y Dionys Mascolo, en especial- que nos permiten asomarnos a esta reconstrucción de la vida desde la perspectiva del otro. Considerar este período arroja una luz nueva para la comprensión de un texto clave de la literatura concentracionaria.
\end{abstract}

Palabras clave: Robert Antelme, literatura concentracionaria, testimonio.

\section{The delirium of words: Robert Antelme's return to life}

\begin{abstract}
In May 1945, after the liberation of Dachau, Robert Antelme, a member of the French Resistance, began his complex process of coming back to life. Two years later, he published his only book, L'espèce humaine, in which he recounted his experience in the camp. Henceforth, he didn't speak nor write about his past, he didn't do interviews, and no biography has been published. This paper takes into account the immediate aftermath of his return during which, on the contrary, he was assailed by the «delirium of words». The uniqueness of this case resides in the evidence provided by his family and friends -specially Marguerite Duras and Dionys Mascolo- that enable us to consider his life reconstruction from the perspective of the other. Considering this period of time casts fresh light on the understanding of a key literary text on the Holocaust Literature.
\end{abstract}

Keywords: Robert Antelme, Holocaust Literature, testimony. 
La historia de Robert Antelme es una historia compleja, llena de acontecimientos, sucesos, amistades, personajes, muchos de ellos claves en la historia intelectual francesa del siglo XX. Intentar comprender y entrelazar todas estas piezas no es una tarea fácil. Antelme no es un autor muy conocido en España, aunque escribió L'espèce humaine, una de las obras esenciales de la llamada literatura concentracionaria. En Francia, a pesar de su temprana publicación (1947) y de lograr once años más tarde ser reeditada por la gran editorial Gallimard, tuvo durante décadas, como muchos otros testimonios, un número bastante reducido de lectores. En 1953, el poeta y editor Jean Cayrol -conocido por ser el autor del guion de la película documental Nuit et brouillard de Alain Resnais- escribía que si el público francés quería documentarse de verdad sobre los campos debía, junto a David Rousset o Germaine Tillion, acercarse a la magnífica y ya olvidada obra de Antelme (Cayrol 1953: 577). Resulta inevitable recordar aquí las palabras de Primo Levi que en 1975 -casi veinte años después de haber publicado Se questo è un uomo- se presentaba a sí mismo como un «autore sfiduciato di un libro che a me sembrava bello, ma che nessuno leggeva» (Levi 1997: 897). Largo y complejo fue el camino que llevó a muchos supervivientes a decidir primero si relatar o no el sufrimiento padecido y cómo surgió después el problema de la recepción y el rechazo que muchos padecieron al finalizar la guerra ${ }^{1}$.

El objetivo de este artículo es el análisis de un período muy concreto de la historia de Antelme. En la mayoría de los testimonios de que disponemos sobre el universo concentracionario nazi, el relato de la experiencia vivida, ya sea en Auschwitz, en Buchenwald o en Dachau, se detiene en el momento en el que el deportado vuelve a casa -como sucede en la obra de Levi o en la novela Sin destino de Imre Kertész- o como en L'écriture ou la vie de Jorge Semprún e incluso en L'espèce humai$n e$, con la apertura y liberación de los campos. Ahí se acaba la narración. No sabemos en la mayoría de los casos lo que ocurrió después, cómo fue el lento proceso del retorno a la vida $-\mathrm{y}$ si este fue finalmente posible-, qué se encontraron al volver a casa o cómo surgió la necesidad de hablar y de dar testimonio. Además, normalmente encontramos tan solo una voz, la del protagonista, que cuenta su propia experiencia, su pasado reciente, y no contamos con la perspectiva del otro o de los otros, del entorno más próximo del deportado que asiste y presencia el retorno del ser querido. El caso de Antelme es muy diferente y quizás único. En primer lugar, Antelme, y este es un punto importante, solo escribió un libro sobre su experiencia en Gandersheim, uno de los subcampos dependientes de Buchenwald donde será deportado y donde permanecerá, como veremos, hasta su liberación en mayo del 45 . A excepción de una serie de artículos publicados poco después de la guerra $-\mathrm{y}$ recogidos ahora en un

${ }^{1}$ Para entender el caso francés, se pueden consultar algunos de los textos más significativos de la historiadora francesa Annette Wieviorka, (2002): L'ère du témoin. Paris: Hachette Littératures; (2003): Déportation et génocide. Entre la mémoire et l'oubli. Paris: Hachette [En especial el segundo capítulo: «Le premiers témoignages»] o el capítulo (1996): «La reconstruction de la mémoire de la déportation et du génocide en France1943-5», en Momigliano Levi, Paolo (ed.): Storia e memoria della deportazione: modelli di ricerca e di comunicazione in Italia ed in Francia, pp. 27-49. Firenze: La Giuntina. 
volumen publicado por Gallimard bajo el título Robert Antelme. Textes inédits sur L'espèce humaine. Essais et témoignages-, Antelme no volvió a hablar ni a escribir más sobre su pasado; no concedió ninguna entrevista. Tampoco se ha publicado además ninguna biografía sobre su vida. Para el estudioso Alain Parrau, la deportación había alterado radicalmente su deseo de escribir, como si su experiencia y el relato de la misma hubieran definitivamente absorbido o quizás cumplido con dicho anhelo (Parrau 1995: 297). De hecho, ya antes de la guerra, había publicado cuatro poemas en un número de la revista Littérature. A su amigo Dionys Mascolo, un personaje importante como veremos en esta historia, le confesaría que había intentado volver a escribir, y que sufría por no lograrlo, pero que había renunciado a ello «parce que cela lui semblait dérisoire» (Antelme 2006: 267) ${ }^{2}$.

En segundo lugar, y no menos importante, una de las particularidades más relevantes en Antelme reside en la multiplicidad de voces que intervienen en su historia, y que ayudan a construirla. Gracias a lo que dijeron y escribieron sus amigos, y a su pareja de aquel entonces, la escritora Marguerite Duras, podemos adentrarnos en esas semanas en las que, tras el regreso, se debatió entre la vida y la muerte y en las que nunca dejó de relatar lo que le había sucedido. «Comment ont-ils retrouvé la vie? Qu'ont-ils rapporté de l'enfer?», se preguntaba, al hablar de los deportados, André Malraux en su obra Antimémoires. Y añadía: «Maints déportés, dans toute 1'Europe, on écrit leurs souvenirs; leur retour à l'humanité n'y figure guère. Il n'est pas facile à un plongeur de rapporter du fond de l'eau ce qu'il y trouve sans le connaître» (Malraux 1967: 592).

Según el historiador Alessandro Portelli todo movimiento de escritura posee unos orígenes; existe una «prehistoria» de lo escrito, una fase incontrolable entre el acontecimiento y su escritura (Portelli 2007: 15). Portelli defiende la idea de que la mayor parte de la memoria escrita no es otra cosa que un barniz que se aplica sobre una oralidad subyacente. Todo escrito está casi siempre precedido de un relato oral. En el caso de Antelme, me gustaría detenerme, en primer lugar, en presentar brevemente su vida, en el contexto que le llevó a la deportación, para más adelante centrarme en el relato a varias voces de su «retorno a la humanidad».

Podríamos decir que toda esta historia nace en una calle, en un lugar preciso: en el número cinco de la calle Saint-Benoît de París, a escasos pasos de la iglesia de Saint-Germain-des-Près, de los famosos Café de Flore o Les deux Magots: «On avait vingt-cinq ans, vingt-sept ans -dirá Marguerite Duras en un documental en

\footnotetext{
${ }^{2}$ Y lo explicaba de esta forma: «La relation qu'il avait faite de quelque chose qui dépasse l'imagination, comme il le dit lui-même, il le dit en préambule à L'espèce humaine-«Ce que nous avons vécu, à nous le remémorer nous semble à nous-mêmes inimaginable»- fait que recourir ensuite à l'imagination lui a sûrement semblé dérisoire. C'est vraisemblablement pour cela qu'il a alors renoncé à poursuivre ce qu'il avait ébauché plus tard» (Antelme 2006: 267). El primer año de su jubilación -Robert Antelme trabajó, entre otras colaboraciones, como lector para la Encyclopédie de la Pléiade, dirigida por Raymond Queneau, hasta 1981- le confiaría a su otro amigo Jean-Louis Schefer su persistente imposibilidad de escribir: «Vous écrivez, Jean-Louis, moi je ne peux plus; ma seule façon d'être ailleurs, c'est de prendre le train. Je prends des trains, c'est presque (sourire) la même chose» (Antelme 2006: 279-280).
} 
1992- [...] On avait trouvé un appartement là, rue Saint-Benoît. C'est là que tout ce qui est arrivé de décisif dans notre jeunesse, dans nos sentiments, dans nos croyances... C'est là que c'est arrivé» (Mascolo y Turine 2002). «On avait», recuerda Duras; se refiere a ella y a Robert Antelme. Estamos en 1942, en el París ocupado por los nazis.

Antelme había nacido en 1917 en el sur de Córcega. Su madre provenía de una familia de la alta burguesía y su padre era subprefecto de policía. Tenía dos hermanas, Alice y Marie-Louise. En 1936 se matricula en la Facultad de Derecho de París y, a través de un amigo en común, conoce a Marguerite Duras. Tres años más tarde, apenas dos semanas después de la invasión alemana de Polonia, se casarán en París. Antelme había sido llamado a filas un año antes, y estaba destinado en la ciudad de Rouen. Conseguirá un permiso de tres días, aunque la misma noche de bodas tendrá que volver al campamento.

Pero su matrimonio, que durará hasta 1947, no será un matrimonio al uso. Era, podríamos decir, un matrimonio abierto. Dionys Mascolo recordaba con estas palabras dicha unión: «[...] ils s'étaient mariés en hâte au moment de la déclaration de la guerre. Ils ne formaient pas un couple. L'un et l'autre avaient des liaisons extérieures. Mais ils avaient l'un pour l'autre une amitié, une admiration sans bornes» (Antelme 2006: 254). En 1941, Marguerite Duras se quedará embarazada, pero el niño nacerá muerto.

Un año después, Mascolo y Duras se conocen en la editorial Gallimard, donde el primero había empezado a trabajar poco tiempo antes. Empiezan una relación que durará hasta 1956, año de su divorcio (se casarán en 1947 y tendrán un hijo ese mismo año). Pero todavía estamos en 1942. Mascolo recordará como si de una instantánea fotográfica se tratara el momento en el que se encontró con Antelme, seis meses después de conocer a Marguerite, al lado del Bon Marché (uno de los más famosos grandes almacenes de la capital francesa). Marguerite había insistido en que se ambos se conocieran. Y desde el principio se convierten en íntimos amigos, una amistad que durará toda su vida. De hecho, muchos de los datos que sabemos de la vida de Antelme se los debemos a Mascolo. Antelme era para su amigo el perfecto «flâneur, au sens baudelairien, un flâneur voyer» (Antelme 2006: 254-255), y así lo fue hasta el final de sus días. En el París de principios de los años 40, los tres, Duras, Antelme y Mascolo, formarían, según las palabras de este último, una especie de «sorte de trio très intime» (Mascolo y Turine 2002), cuya amistad y compromiso político los llevaría conjuntamente a entrar a la resistencia.

Todos ellos se unen entonces, en otoño del 43, al que pasaría a llamarse más tarde Mouvement National des Prisonniers de Guerre et Déportés que dirigía François Mitterrand. Antelme había llevado a cabo ya antes pequeñas actividades de resistencia en la prefactura de París, donde había trabajado como redactor, destruyendo denuncias, ayudando a extranjeros, filtrando documentos. Es a través de un amigo común de Antelme y Mitterrand, como Mascolo, Duras y el protagonista de esta historia entran juntos en la resistencia. En el apartamento de la calle SaintBenoît, según Egdar Morin, filósofo y sociólogo francés, se produce un «phénomène d'atraction et de satellisation» (Mascolo y Turine 2002) de gente muy distinta, creándose un lugar de fraternidad y aprendizaje en el que amigos y conocidos dis- 
cutían y hablaban de literatura y de política. Entre este círculo de amistades que se construye en aquel momento aparecerán nombres fundamentales de la historia y del mundo intelectual francés del siglo XX. Se había fundado, según Mascolo, más que un grupo, «une sorte de communauté, [...] très espontanée. Elle répondait à un besoin... négatif. Nous étions en permanence scandalisés par l'état du monde» (Mascolo y Turine 2002). Al rememorar las motivaciones que le habían llevado a entrar en la Resistencia, Mascolo admite que en su recuerdo sobresale el «goût de l'aventure» (Mascolo y Turine 2002), una aventura más individual que metafísica, moral o política. Mitterrand, por su parte, recordaba a Antelme como un hombre que no era en sí un hombre de acción, que no habría ido solo en búsqueda de un grupo de la Resistencia, sino que el destino quiso que estuviera en contacto cotidiano con miembros de esta última (Antelme 2006: 257). «On n'a pas été des héros.», apuntaba Marguerite Duras casi cincuenta años más tarde, «La Résistance este venue à nous parce qu'on était d'honnêtes gens. Je pense que François [Miterrand] a eu cette impression que Robert, Marie-Louise [Antelme] et moi, on était des gens à qui on pouvait se confier» (Antelme 2006: 258).

El 1 de junio de 1944, tras una emboscada, Antelme es arrestado en París por la Gestapo. Es internado primero en el campo de Fresnes, rápidamente deportado a Buchenwald y de ahí transportado al subcampo de Gandersheim. También es arrestada su hermana Marie-Louise, que será deportada a Auschwitz, de donde no volverá con vida. Antelme permanecerá en Gandersheim hasta abril del 45. Es entonces, ante la llegada de los aliados, cuando las SS trasladan, en una de las llamadas marchas de la muerte, a los prisioneros aún con vida al campo de Dachau, que finalmente será liberado por el ejército americano. Y es ahí, en Dachau, donde de nuevo aparece el nombre de François Mitterrand en mayo de 1945 y donde empieza el relato del retorno a la vida de Robert Antelme.

Mitterrand había sido nombrado por el gobierno provisional Subsecretario de Estado para los refugiados, prisioneros y deportados. El general De Gaulle lo envía junto al general Lewis para participar en nombre de Francia en la apertura de algunos campos, entre ellos Dachau. Según relata el propio Mitterrand, la llegada a Dachau fue un espectáculo «tragique et inoubliable» (Antelme 2006: 262). Los cadáveres se amontonaban por todas partes. Los hornos crematorios seguían funcionando. Empiezan a caminar por el campo, él y otro de sus camaradas del Movimiento, y de repente oyen una voz que dice su nombre: «François». En un primer momento no lo reconocen, pero se trataba de Robert Antelme. La última vez que se habían visto era casi un año antes, en junio del 44. Rápidamente Mitterrand le pide al general Lewis la autorización para llevárselo a París. La respuesta es negativa porque la posible amenaza de tifus impedía sacar a cualquier deportado del lugar. Al llegar a París, Mitterrand contacta con Mascolo y Duras y les comunica que Antelme sigue con vida, pero que parece condenado a morir. Todo sucede entonces muy rápido y es aquí donde a los recuerdos de Mitterrand se suman los de Marguerite Duras:

Je ne sais plus quel jour c'était, si c'était encore un jour d'avril, non c'était un jour de mai, un matin à onze heures le téléphone a sonné. Ça venait d'Allemagne, c'était François Morland [alias de Mitterrand]. Il ne dit pas bonjour, il est presque brutal, 
clair comme toujours. «Écoutez-moi. Robert est vivant. Calmez-vous. Oui. Il est à Dachau. Écoutez encore de toutes vos forces. Robert est très faible, à un point que vous ne pouvez pas imaginer. Je ne dois vous le dire: c'est une question d'heures. Il peut vivre encore trois jours, mais pas plus. (Duras 2007: 65)

En 1985 la escritora francesa publicará un volumen de relatos titulado La douleur. El primero de ellos, que lleva el mismo título, se centra en el relato de la espera y del retorno a la vida de Antelme. No conozco ningún texto igual, una obra en la que podamos conocer el punto de vista del otro; en este caso el de la mujer que espera la vuelta del marido del campo. Se ha escrito y discutido mucho sobre su veracidad, sobre la génesis de su publicación, sobre su carácter ficcional y sus reescrituras ${ }^{3}$. El estatuto del texto es complejo. En el prólogo, Marguerite Duras presenta su relato como un diario, como un relato autobiográfico: «J'ai retrouvé -escribe- ce Journal dans deux cahiers des armoires bleues de Neaphle-le-Château. Je n'ai aucun souvenir de l'avoir écrit» (Duras 2007: 12). Dentro del cuadro ficticio del diario íntimo olvidado, explica Marie Bornand, la legitimidad de su testimonio reposa en la voz protagonista que narra la historia en primera persona, y que coincide con el nombre de la autora. Se trata de un caso único de testimonio indirecto, concebido y reelaborado, cuarenta años más tarde, como respuesta al texto de Antelme (Bornand 2004: 135) ${ }^{4}$. Novela o no, diario o no, o «artefacto reescrito» según Philippe Lejeune (Lejeune 1998: 317 apud Wroblewski 2010: 64), es un relato que atrapa al lector desde las primeras páginas.

Con la llamada de Mitterrand cambia la estructura del texto, se interrumpe la forma clásica del diario - cada día del mes, una nueva entrada- y la sucesión de días desaparece. Mascolo y su amigo Georges Beauchamp deciden entonces coger un coche y, provistos de documentación falsa, salen de París en dirección a Dachau. En el año 1987, Mascolo publicará el libro Autour d'un effort de mémoire: sur une lettre de Robert Antelme, en el que recordará con detalle dicho viaje y el retorno a la vida de su amigo. Haciéndose pasar por agentes del servicio de información, logran entrar en el campo: «Je ne sais -recuerda-combien de temps j'ai erré dans les allées, entre les blocks, scrutant les visages, questionnant ceux qui peuvent parler» (Mascolo 1987: 48). De repente, como le había sucedido a Mitterrand, escucha una voz pronunciar su nombre: «Je reconnais Robert à l'espace qui sépare ses incisives supérieures» (Mascolo 1987: 48) ${ }^{5}$. Hacen creer a los americanos que Antelme dis-

\footnotetext{
3 Véase, por ejemplo, el artículo de Ania Wroblewski (2010).

4 La reacción de Antelme a la publicación de la obra de Duras fue muy dura. En el año 2009, Monique Antelme -su segunda mujer- recordaría el golpe que supuso La douleur para Antelme: «Je comprends que Robert ait été blessé. Il m'a dit qu'elle savait très bien, mieux que toi, il me disait, que lui n'aurait pas supporté ça, donc elle n'aurait pas du le faire sachant sa réaction, à lui. Il m'a dit qu'il ne supportait pas qu'elle parle de sa déportation et qu'elle en parle de cette manière-là. Il y avait les deux choses, mais aussi qu'elle parle tout simplement, il trouve que c'est sujet qu'elle n'aurait pas dû aborder étant donné qu'il lui avait quand même beaucoup parlé en rentrant et tout ça, qu'elle savait l'importance... C'était capital évidemment, pour Robert, sa déportation» (Adler 2009). Antelme prometió no volver a dirigirle la palabra a Marguerite Duras.

5 Marguerite Duras, sin embargo, cuenta en La douleur que fue el camarada de Mitterrand el que reconoció a Antelme por sus dientes, y no Mascolo (Duras 2007: 66).
} 
ponía de informaciones sobre los servicios de la Gestapo todavía activos en Francia, y logran así sacarle del campo y llevarlo, en un viaje que duró dos días, de vuelta a casa. «Je ne puis relater -explica- ce qui suit qu'au prix d'un effort analogue à celui qu'exigent les récits de rêves» (Mascolo 1987: 49).

Antelme está moribundo. Después de apenas setenta kilómetros de viaje, cuando el peligro parece ya lejano y después de que desaparezca su miedo a volver a ser detenido, empezará a hablar y no dejará de hacerlo en todo el trayecto:

Il parle. Il dit tout. [...] Dans son épuisement physique, il n'est plus que parole. Je n'ai pas à le questionner. Il dit tout. Tout ce qu'il a vécu depuis un an, épisode par épisode, sans ordre, l'un évoquant l'autre. Garder le silence plus de quelques instants lui serait impossible. Il parle continûment. Sans heurt, sans éclat, comme sous la pression d'une source constante possédé du besoin véritablement inépuisable d'en avoir dit le plus possible avant de peut-être mourir, et la mort même n'avait manifestement plus d'importance pour lui qu'en raison de cette urgence de tout dire qu'elle imposait. (Mascolo 1987: 50)

Antelme pesa $35 \mathrm{~kg}$, es un hombre que no pertenece ya a ningún lugar, ni a ningún limbo; es un ser abandonado entre dos mundos. Pero antes de morir, debe compartir lo que ha vivido. Mascolo admite no recordar con exactitud las palabras de su amigo, aunque sabe que se grabaron en su memoria, y que hubiera sido capaz de recuperarlas y de compartirlas si Antelme hubiera muerto. Lo mismo le ocurre cuando intenta hacer el esfuerzo de recordar su aspecto. No es capaz de hacerlo, pero conserva la imagen del impacto y del silencio que su figura produjo cuando, al día siguiente de abandonar Dachau, entraron en una cervecería de Verdún:

A notre entrée, les conversations les plus proches s'interrompent, et la vague de silence gagne bientôt tout la salle. De table en table, on se lève alors à mesure que nous avançons. Le silence, l'immobilité, complète, dureront jusqu'après que nous aurons eu trouvé place. [...] rien ne fut dit, de part ni d'autre. Une telle manifestation spontanée d'émotion collective, d'une intensité qui n'est comparable qu'à celle de certains rêves métaphysiques, je n'en connais pas d'exemple aussi pur. (Mascolo 1987: 55)

Antes de abandonar la ciudad, visitan a un médico que les recomienda no darle de comer, su cuerpo está demasiado débil y el corazón podría fallar. Pero Antelme sigue hablando, sentado en el asiento de atrás del coche, junto a su amigo. Al día siguiente llegan a París. A primera hora de la tarde, Marguerite Duras los estará esperando, asomada desde su ventana. Mascolo recuerda con precisión este momento. La escritora los ve, baja a su encuentro pero enseguida huye, y sube corriendo las escaleras. Permanecerá durante mucho tiempo sola, escondida tras su ropa, en un armario de una habitación.

Durante tres semanas, Antelme se debatirá entre la vida y la muerte. La fiebre y la disentería se apoderan de su cuerpo. En La douleur, podemos leer el relato de los diecisiete días en los que luchará por seguir con vida. Duras describe con dureza y con muchos detalles ese período de sus vidas. Cada vez que, por ejemplo, Antelme abandonaba la habitación, esta... 
s'emplissait d'une odeur qui n'était pas celle de la putréfaction, du cadavre - y avaitil d'ailleurs encore dans son corps matière à cadavre - mais plutôt celle d'un humus végétal, l'odeur des feuilles mortes, celle des sous-bois trop épais. C'était là en effet une odeur sombre, épaisse comme le reflet de cette nuit épaisse de laquelle il émergeait et que nous ne connaîtrions jamais. (Duras 2007: 74)

No dejará, sin embargo, de hablar, como lo había hecho en el viaje de regreso a París. Y de repente, una mañana, la fiebre desaparece y exclama: «J'ai faim» (Duras 2007: 75). Y todo cambia, aunque el regreso a la vida fue largo y complejo. El hambre se apodera enseguida de él y se vuelve insaciable. Roba pan a escondidas, se enfada si la comida no se le sirve rápidamente, recoge las migas de pan que se caen en su pantalón y en el suelo. Un mes después de su llegada, lo trasladan a una casa de reposo para deportados y le comunican que su hermana Marie-Louise ha muerto en Auschwitz. Poco después, Duras le anuncia su intención de divorciarse. Empieza a recuperar su peso, pero debe caminar con un bastón. Año y medio más tarde, se quedaba aún de vez en cuando largos ratos sin hablar, en silencio, mirando fijamente el suelo.

El 21 de junio de 1945 le escribe una carta a Mascolo, un texto importante, el primer acto que realiza, en palabras de Antelme, como «vivant «solidifié»»» (Mascolo 1987: 13). Pasados cuarenta años, estando aún Antelme con vida -en 1983 sufrió un ictus que lo dejó sin habla e inmovilizado en un hospital durante siete años-, Mascolo decide publicarla. Fue una decisión difícil ${ }^{6}$. En la carta, Antelme le confía las dificultades que está viviendo:

[...] pendant les premiers jours où j'étais dans mon lit et je vous ai parlé, à toi et à Marguerite surtout, je n'étais pas un homme de la terre. J'insiste sur ce fait qui me hante rétrospectivement. D'avoir pu libérer des mots qui étaient à peine formés et en tout cas n'avaient pas de vieillesse, n'avaient pas d'âge, mais se modelaient seulement sur mon souffle, cela vois-tu, ce bonheur m'a définitivement blessé et à ce moment-là, moi qui me croyais si loin de la mort par le mal -typhus, fièvre, etc.- je n'ai pensé mourir que de ce bonheur. Et maintenant je recommence à donner une forme aux choses [...]. (Mascolo 1987: 14)

La felicidad de haber podido hablar le ha herido. Surge, además, el problema de volver a la vida, de retornar de ese otro mundo infernal, y de reconocerse o no en el ser que una vez fue. Antelme afirma sentirse un recién nacido y relata la dificultad de comenzar de nuevo a parecerse a su antiguo yo: «Tous mes amis m'accablent avec une satisfaction pleine de bonté, de ma ressemblance avec moi-même, et il me semble que je vis à l'envers le Portrait de Dorian Gray» (Mascolo 1987: 16-17).Y concluye esta carta con las siguientes palabras:

Il me reste encore parfois un sentiment trop vif de l'horreur, mais sans doute bientôt tout cela sera-t-il aplani, neutralisé. Alors peut-être j'accepterai la ressemblance avec

\footnotetext{
${ }^{6}$ Véanse los motivos y las reservas de Mascolo al principio de su libro, y la correspondencia con Maurice Blanchot incluida en el mismo (Mascolo 1987: 5-7 y 75-88).
} 
moi-même parce que je saurai qu'elle n'est pas; j'accepterai le portrait : il n'y aura plus de portrait. (Mascolo 1987: 17)

La «véritable hémorragie d'expression» que invadió a muchos deportados tras su regreso a casa, expresaba, según él, las siguientes verdades: «que chacun veut mettre toute sa persévérance à se reconnaître dans ce temps passé et que chacun veut que l'on sache que c'est bien le même homme, celui qui parle et celui qui était là-bas» (Antelme 2006: 44). Está claro, explica, que se trata del mismo hombre; toda esta reflexión puede parecer por tanto superflua. El problema para él radica no en reconocer que nos encontramos ante el mismo hombre, sino en reconocer que este hombre pueda hablar en calidad de deportado: «On ne discute pas - concluye- sur l'aller et retour, on discute sur le bagage qui accompagne le retour» (Antelme 2006: 45).

A pesar de la incomprensión y de la falta de escucha a las que muchos deportados tuvieron que hacer frente, muchos de ellos continuaron hablando, intentando, como Antelme, mostrar lo que había pasado, explicando, comentando sus experiencias. «Il y a deux ans, -escribe en el prólogo de L'espèce humaine-durant les premiers jours qui ont suivi notre retour, nous avons été, je pense, en proie à un véritable délire. Nous voulions parler, être entendus enfin» (Antelme 2007: 9). En poco tiempo redactará su obra y la publicará en 1947 en la editorial La Cité Universelle, que había creado con Marguerite Duras en el piso de la calle Saint-Benoît. Diez años después, logrará que Gallimard vuelva a publicar su libro, un caso muy similar al de las dificultades que tuvo Primo Levi lograr que la editorial Einaudi publicara Se questo è un uomo.

Comprender el «delirio de la palabra» de Antelme y la escritura de L'espèce humaine exige tomar en consideración, aunque sea muy brevemente, otra historia dentro de la misma historia. En 1946, Mascolo y Antelme se inscriben en el partido comunista francés, aunque su compromiso y militancia es compleja. En el caso de Antelme, su militancia en el PCF durará poco, puesto que apenas cuatro años después fue expulsado del partido. Desde el principio, manifiesta activamente su desacuerdo profundo con la política cultural del partido, en especial con el papel que deben jugar la literatura y la estética en relación con la lucha revolucionaria. Antelme y otros proponen la libre creación y discuten sobre el rol que el intelectual comunista debe tener, su relación con el partido y la importancia de la independencia de la creación literaria. Pero a pesar de su ruptura, el compromiso intelectual y político de Antelme recorre toda L'espèce humaine; es un reflejo de su visión del mundo, y modela o configura su visión del campo. Para él, los campos de concentración y de exterminio nazi representan, como dirá también otro deportado francés, David Rousset, la culminación de la deshumanización propia del capitalismo. El régimen concentracionario, como señala Martin $\mathrm{Crowley}^{7}$, es concebido como la forma exacerbada del capitalismo, en el que el deportado se convierte en proletario (Crowley

${ }^{7}$ Martin Crowley, uno de los autores que más ha trabajado sobre Antelme, escribe que al leer L'espèce humaine claramente se reconoce que el libro ha sido escrito por un militante comunista (Crowley 2004: 92). 
2004: 73). Ambos luchan por resistir, por seguir con vida. En el campo, como en la historia, se juega la liberación de la humanidad, y el opresor está condenado al fracaso. La idea fundamental que recorre toda la obra es la reivindicación de la pertenencia a la especie humana. Ante el intento por parte del sistema nazi de destruir la humanidad, de deshumanizar a sus víctimas, el deportado debe luchar por su vida, por sobrevivir. Así, no dejarse morir se entiende como un deber de militancia del prisionero político. Y esta lucha, como escribía Georges Perec en un breve texto titulado «Robert Antelme ou la vérité de la littérature», es ante todo un fenómeno de conciencia, «une prise de conscience de son corps comme totalité irréductible, une découverte de sois comme singularité indestructible» (Perec 1992: 104). En el prólogo de su obra, Antelme escribía las siguientes palabras: «Le ressort de notre lutte n'aura été que la revendication forcenée, et presque toujours elle-même solitaire, de rester, jusqu'au bout, des hommes» (Antelme 2007: 11). Las SS pueden matar a un hombre, «mais il ne peut pas le changer en autre chose» (Antelme 2007: 241). Ellos mismos pertenecen a la misma especie y a la misma historia. Finalmente, en el infierno del campo, lo que queda del hombre son la posibilidad y la fuerza de resistir.

Quisiera acabar mi texto subrayando, como lo ha hecho Alain Parrau, que una de las particularidades más relevantes de Antelme es la belleza de su escritura junto a la agudeza de su pensamiento (Parrau 1995: 9). Fue capaz de ir más allá del testimonio y crear una obra literaria, algo que muy pocos textos han logrado. Como afirmó Perec, con L'espèce humaine volvió a nacer la literatura, una literatura que en aquellos años había perdido su poder. A través de la transformación de una experiencia tan extrema en lenguaje, y con las dificultades que esto conllevó, Antelme logró convertirse en el ejemplo más perfecto de lo que podía y debía ser la literatura. «Par son mouvement -concluía Perec- par sa méthode, par son contenu, enfin, L'Espèce humaine définit la vérité de la littérature et la vérité du monde» (Perec 1992: 114).

\section{Referencias bibliográficas:}

ADLER, Laure (2009): «Hommage à Monique Antelme», France Culture; redifusión del programa Hors Champs, el 24 de octubre de 2012, de la entrevista realizada por Laure Adler a Monique Antelme dentro del programa Avec Duras, en agosto del año 2009. Transcripción de la entrevista realizada por Taos Aït Si Slimane. [en línea]. En: http://www.fabriquedesens.net/Hors-champs-Monique-Antelme [07/05/2016].

AnTELME, Robert (2006): Textes inédits sur L'espèce humaine. Essais et témoignages. Paris: Gallimard [1996].

- (2007): L'espèce humaine. Édition revue et corrigée. Paris: Gallimard [1947].

BORNAND, Marie (2004): Témoignage et fiction. Paris: Droz.

CAYROL, Jean (1953): «Témoignage et littérature», Esprit 21: 575-578.

CROWLeY, Martin (2004): Robert Antelme. L'humanité irréductible. Préface de Edgar

Morin. Paris: Lignes-Éditions Léo Scheer.

DURAS, Marguerite (2007): La douleur. Paris: P.O.L [1985]. 
LEVI, Primo (1997): «Il sistema periodico», en Opere, vol. I, edizione di Marco Belpoliti; introduzione di Daniele Del Giudice. Torino: Einaudi [1975].

MalrauX, André (1967): Antimémoires, Tome 1. Paris: Gallimard.

MASCOLO, Dionys (1987): Autour d'un effort de mémoire: sur une lettre de Robert Antelme. Paris: M. Nadeau.

Mascolo, Jean y TURINe, Jean-Marc (dirs.) (2002): Autour du groupe de la rue SaintBenoît de 1942 à 1964. L'esprit d'insoumission. DVD [Cinta documental]. Paris: BenoîtJacob [1992] [en línea]. En: www.youtube.com/watch?v=vDhN8NLcZ7E [05/06/2016].

PARRAU, Alain (1995): Écrire les camps. Paris: Belin.

PEREC, Georges (1992): L.G. Une aventure des années soixante. Paris: Éditions du Seuil.

PORTELLI, Alessandro (2007): Storie orali: racconto, immaginazione, dialogo; introduzione di Ronald Grele. Roma: Donzelli.

WrobleWSKI, Ania (2010): «Réécrire, revivre, oublier: la genèse et la publication de "La douleur"». Interférences littéraires, 4 («Indicible et littérarité»): 63-74 [en línea]. En: http://www.interferenceslitteraires.be/node/80 [17/09/2012]. 